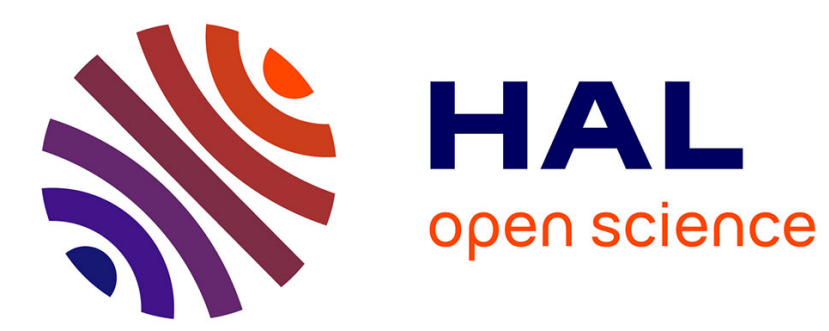

\title{
High speed closed loop control of a dielectrophoresis-based system.
}

Mohamed Kharboutly, Michaël Gauthier

\section{To cite this version:}

Mohamed Kharboutly, Michaël Gauthier. High speed closed loop control of a dielectrophoresis-based system.. IEEE International Conference on Robotics and Automation, ICRA 2013., Jan 2013, Germany. pp.1438-1443. hal-00834100

\section{HAL Id: hal-00834100 https://hal.science/hal-00834100}

Submitted on 14 Jun 2013

HAL is a multi-disciplinary open access archive for the deposit and dissemination of scientific research documents, whether they are published or not. The documents may come from teaching and research institutions in France or abroad, or from public or private research centers.
L'archive ouverte pluridisciplinaire HAL, est destinée au dépôt et à la diffusion de documents scientifiques de niveau recherche, publiés ou non, émanant des établissements d'enseignement et de recherche français ou étrangers, des laboratoires publics ou privés. 


\title{
High speed closed loop control of a dielectrophoresis-based system
}

\author{
Mohamed Kharboutly $^{1}$, Michaël Gauthier ${ }^{1}$, Member, IEEE,
}

\begin{abstract}
Nanosciences have recently proposed a lot of proofs of concept of innovative nanocomponents and especially nanosensors. Going from the current proofs of concept on this scale to reliable industrial systems requires the emergence of a new generation of manufacturing methods able to move, position and sort micro-nano-components. We propose to develop 'No Weight Robots-NWR' that use non-contact transmission of movement (e.g. dielectrophoresis, magnetophoresis) to manipulate micro-nano-objects which could enable simultaneous high throughput and high precision. This article deals with a control methods which enables to follow a high speed trajectory based on visual servoing. The non-linear direct model of the NWR is introduced and the calculation of the inverted model is described. This inverted model is used in the control law to determine the control parameter in function of the reference trajectory. The method proposed has been validated on an experimental setup whose time calculation has been optimized to reach a control period of $1 \mathrm{~ms}$. Future works will be done on the study of smaller components e.g. nanowires, in order to provide high speed and reliable assembly methods for nanosystems.
\end{abstract}

\section{INTRODUCTION}

This article deals with the closed loop control of a noncontact dielectrophoresis system which can be considered as an original robotic structure compared to the current industrial robot. The first industrial robot UNIMATE [1] based on standard joints was commercialized in 1961 (see figure 1). Nowadays more than one million of robots are in use all over the world. In the 1980's the use of compliant structures in robotics [2] was started to enable high precision positioning making them, at present, the most widely used structure for microscale robots [3], [4]. However, transmission of movement in such robots is obtained via the movements of mechanical parts which largely limits throughput due to inertial effects. In the 2000's, LightWeight Robots [5], [6] have been developed by KUKA[7] to reduce robot inertia. However, the impact of inertia is still important in the small scales (micro-nano) where the inertia of the object is highly negligible compared to the one of the robots. A new consist in developing robots that use non-contact transmission of movement to manipulate micro-nano-objects [8], [9], [10]. Besides eliminating the inertia of a robotic structure, this approach also eliminates friction and adhesion (between the tweezer and the component) which highly reduce robot performance and life time.

These 'No Weight Robots' NWR are at the cross-road between parallel robot and current non-contact manipulation.

1 FEMTO-ST Institute, AS2M department, Université de FrancheComté/CNRS/ENSMM/UTBM, 24 rue Savary, 25000 Besancon, France.
Firstly, NWR consists of moving components by applying forces coming from several physical field sources which have a similar effect to parallel robotics [11], [12] where the platform is moved by several mechanical forces coming from several robotic legs. The use of non-contact forces, rather than mechanical forces, changes the robot design drastically. In this regard, existing robotic approaches cannot be transferred to NWR. Secondly, current noncontact manipulation has been achieved mostly by open loop for object positioning or self-assembly [13-20] . The only exception concerns laser trapping which has been experimented in closed-loop by Arai et al. [19], [20]. However, laser trapping induces forces around tens of picoNewtons limiting the achievable throughput. The dielectrophoresis proposed in this paper generate forces around thousand times higher [21], [22]. Providing closed loop control strategies will enable active and reprogrammable trajectory control and guarantee the final position of a manipulated object.

This paper introduces a numerical model of a microbead's behaviour in a dielectrophoresis system, in the next section a closed loop control strategy is presented then the experimental setup is presented as well as experimental results.

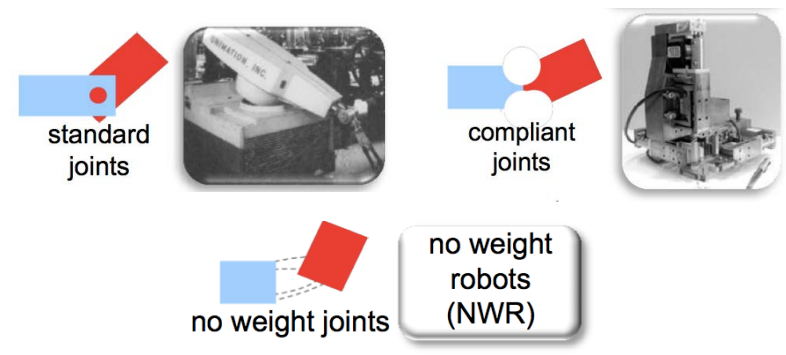

Fig. 1. Movement transmission used in robotics: (i) standard joints used in a majority of robots; (ii) compliant joints based on mechanical deformation used in high precision positionning systems; (iii) the third alternative: movement transmission based on non contact forces

\section{Direct Dynamic Model of A DIELECTROPHORESIS-BASED SYSTEM}

In this section, we present a $3 D$ dynamic model of a bead driven by a dielectrophoretic force. This model is used in the trajectory control law to determine the voltage to applied in function of the trajectory reference. As we consider only beads and no dephasing, electrorotation is not considered. 


\section{A. Dielectrophoresis force similator}

In order to compute the electric field and then the dielectrophoretic force applied to a micro-object in an electrode structure, a numerical simulator is needed. This numerical simulator must be able to compute the dielectrophoretic force generated by very complex geometries in a very short time. For one hand, corresponding analytic equations are very complex and hard to be established. For a second hand, the finite element modeling (FEM) solution is limited to a long computation time and specially when electric voltage changes frequently. Thus, we propose to use the hybrid numeric simulator proposed in [16] gathering the ability of the FEM solution to simulate complex electrodes geometry and the short computation time of the analytical equations. According to [23], the dielectrophoretic force $\vec{F}_{D E P}$ applied to the micro-bead's center $X(x, y, z)$ with respect to the electric field $\vec{E}(X, U)$ can be written as:

$$
\vec{F}_{D E P}(X, U)=2 \pi \epsilon_{m} r^{3} \operatorname{Re}[K(\omega)] \nabla\left(\vec{E}^{2}(X, U)\right),
$$

where

$$
K(\omega)=\frac{\epsilon_{p}^{*}-\epsilon_{m}^{*}}{\epsilon_{p}^{*}+2 \epsilon_{m}^{*}},
$$

and $\epsilon_{p}^{*}$ and $\epsilon_{m}^{*}$ are respectively the complex permittivity of the particle and the medium with:

$$
\epsilon^{*}=\epsilon+j \frac{\sigma}{\omega}
$$

$\epsilon$ is the relative permittivity, $\sigma$ is the conductivity and $\omega$ is the angular velocity of the electric field. Thus, if we consider a configuration of $n$ electrodes, by applying $n-1$ sinusoidal electric voltages identified by there magnitudes $U=\left[U_{1}, \ldots U_{n-1}\right]$ and there angular velocity $\omega$, the electric field $\vec{E}(X, U)$ can be computed using the hybrid method described in [16]. This hybrid method consists in computing the electric field $\vec{E}(X, U)$ by integrating the surface charge density on the electrodes. In fact the electric charge density $Q$ and the magnitudes of the applied voltages $U$ on the electrodes are linearly related:

$$
Q=\sum_{i=1}^{n-1} C_{i} U_{i},
$$

where $U_{i}$ is the magnitude of the applied voltage on the ith electrode and $C_{i}$ is the elementary inter-capacitance between the electrodes influenced by the ith electrode. The inter-capacitance between the electrodes depends only on the geometric shape of the electrodes and the electric permittivity of the medium. The $C_{i}$ is simulated using FEM software. These simulations are executed in preprocessing which reduces the total time of the force computation. If we consider the planar electrodes drawn in the figure 2 (red lines), the number of electrodes $\mathrm{n}$ is equal to 4 and they are placed in the $x, y$ plane.

To compute the electric charge density $Q$ with respect to the applied voltages $U=\left[U_{1}, U_{2}, U_{3}\right], n-1=3$ FEM simulations are required. The figures 2 (a) and 2(b)

show the elementary inter-capacitances $C_{1}$ and $C_{3}$. The figure 2(c) shows how the electric charge density $Q$ is analytically computed with respect to the applied voltages $U=[75 \mathrm{~V}, 0,75 \mathrm{~V}]$ and the elementary inter-capacitances $C_{1}$ and $C_{3}$.

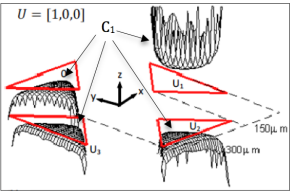

(a) Elementary capacitance $C_{1}$

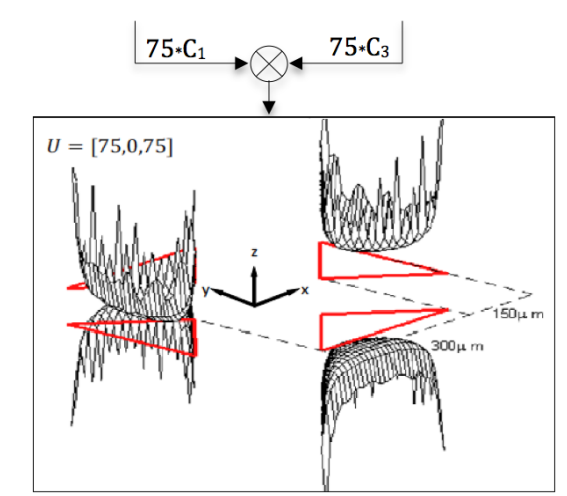

(c) The computed charge density $C=75 \times C_{1}+$ $75 \times C_{3}$.

Fig. 2. The electric charge density computed on the electrodes by applying the following electric voltages: $U=[75 \mathrm{~V}, 0,75 \mathrm{~V}]$.

Once the matrix of the electric charge density $Q$ is computed, the electric field can be calculated analytically in a point $X(x, y, z)$ in the medium. In fact, with each value $Q_{i, j}$ of the computed matrix $Q$ corresponds a $x_{i, j}, y_{i, j}$ point on the electrodes $\left(z_{i, j}=0\right.$ because of the electrodes are in the $x, y$ plane). Thus, the expression of the electric field $\vec{E}$ at the point $X(x, y, z)$ is:

$$
\vec{E}(x, y, z)=\sum_{i}\left(\sum_{j} \frac{Q_{i, j} \vec{r}}{4 \pi \epsilon_{m}\|\vec{r}\|^{3}}\right),
$$

where $r=\left[x-x_{i, j}, y-y_{i, j}, z\right]$, and the DEP force can be also computed analytically with respect to (1). The figure 3 resumes the DEP modeling simulator (DMS) block. The block's inputs are the geometric shape of the electrodes, the applied voltages and the micro-bead's current position. This block generates the computed $x, y$ and $z$ components of the dielectrophoretic force applied to the micro-bead in its center.

\section{B. $3 D$ direct dynamic model}

The inertia of a micro-bead in a dielectrophoretic force field can be neglected [9], [10]:

$$
\vec{F}_{D E P}(X)+\vec{F}_{\text {Drag }}(\dot{X})+\vec{P}=0 .
$$




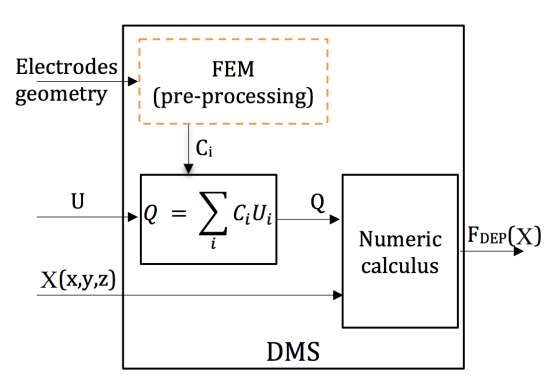

Fig. 3. DEP modeling simulator (DMS).

In the micron scale the Stokes approach of the viscosity friction is valid, $\vec{F}_{\text {Drag }}(\dot{X})$ becomes:

$$
\vec{F}_{D r a g}(\dot{X})=-6 \pi \nu R \overrightarrow{\dot{X}}
$$

where $\nu$ is the dynamic viscosity and $R$ the radius of the micro-bead. The dynamic equation is thus:

$$
\overrightarrow{\dot{X}}=\frac{\vec{F}_{D E P}(X)+\vec{P}}{6 \pi \nu R}=\frac{\vec{F}_{D}(X)}{6 \pi \nu R},
$$

where $\vec{F}_{D}$ is the driving force, sum of the dielectrophoretic force and the weight.

The diagram in the figure 4 illustrates the $3 D$ direct dynamic modelling. Having the applied electric voltages and the electrodes geometry as input, the direct modelling simulator computes the corresponding micro-bead's trajectory. In generally, the micro-bead's behavior in dielectrophoretic force field is characterized by its high dynamics and nonlinearity. This numeric simulator is experimentally validated in [16] where we have shown that the dynamics of the microbead is less than $3 \mathrm{~ms}$. Moreover the behaviour of the microbead is subjected to a high nonlinearity and especially when the micro-bead approaches the electrodes.

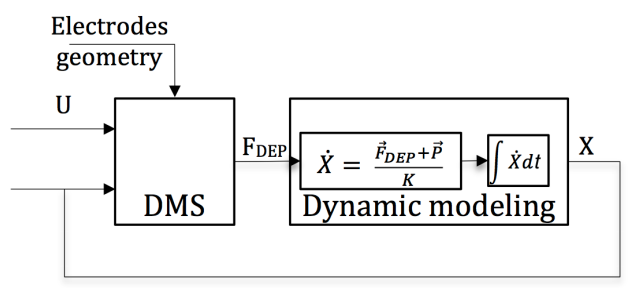

Fig. 4. A dynamic modeling and DMS are used to compute the microbead's 3D trajectory.

\section{HIGH SPEED CONTROL STRATEGY}

As mentioned above, the dielectrophoretic-based system is characterized by the high speed motion of the microobject, which makes it compatible for a high speed control system. Thus to use the dielectrophoretic force to control the position of a micro-object in such system two main aspect must be considered. The first one is the high speed of the micro-object's motion, where the control system must be theoretically at least twice faster than the time constant of the system. The second one is the high non-linearity of the generated force with respect to the applied voltages and the micro-object's position. For these reasons, classic control low, such as PID controler are not efficient. In this study we propose to consider the dielectrophoretic system as a robotic manipulator where the dynamic model presented above is the direct dynamic model (DDM). The inverse dynamic model will be used later to control the micro-object's velocity and position. The computation of the DDM presented in (4), (5) and (8) is quite long and it takes several milliseconds even when using a high-speed computing processor, e.g. if we use a $1 \mathrm{MHz}$ control system, at least $10^{4} \mathrm{CPU}$ clock are needed, witch mean that $10 \mathrm{~ms}$ are required to complete the computation. In orer to imrpove the calculation time in the controler a $3 D$ simplified model has been developed.

\section{A. 3D simplified model}

In order to reduce the complexity of the computation, we will consider that the electrodes surface is planar in the $x, y$ plane. The $3 D$ dielectrophoretic dynamic modeling simulator is designed to run on a classic $P C$ with high performance (typically $\mathrm{GHz}$ ) and it is not optimized to be integrated directly into a controller card with lower calculation performance (typically $\mathrm{MHz}$ ). Thus, a simplification of the $3 \mathrm{D}$ simulator is proposed. We assume that the micro-bead will move only in a limited space above the electrodes surface in a parallelipedic workspace. The simplified 3D DDM (SDDM) uses a similar approach to the $3 D$ DDM presented above. In this SDDM, a database of the elementary spacial force is created. This database links the $3 D$ dielectrophoretic force directly to the applied voltages, which will reduce sufficiently the computation time. Using the linear relationship between the electric field $\vec{E}$ and the applied voltages $U$, the dielectrophoretic force can be written as a second order equation with respect to the electric voltages. Using the electrodes configuration presented in the figure 5, and the following electric voltages vector:

$$
U=\left[u_{1}, u_{2}, u_{3}\right]=\left[V_{1}-V_{4}, V_{2}-V_{4}, V_{3}-V_{4}\right],
$$

the driving force $\left[F_{D_{x}}, F_{D_{y}}, F_{D_{z}}\right]$ can be written as the following:

$$
\begin{aligned}
F_{D_{x}} & =f_{x 1} u_{1}^{2}+f_{x 2} u_{2}^{2}+f_{x 3} u_{3}^{2} \\
& +f_{x 12} u_{1} u_{2}+f_{x 13} u_{1} u_{3}+f_{x 23} u_{2} u_{3} \\
F_{D_{y}} & =f_{y 1} u_{1}^{2}+f_{y 2} u_{2}^{2}+f_{y 3} u_{3}^{2} \\
& +f_{y 12} u_{1} u_{2}+f_{y 13} u_{1} u_{3}+f_{y 23} u_{2} u_{3} \\
F_{D_{z}} & =-m g+f_{z 1} u_{1}^{2}+f_{z 2} u_{2}^{2}+f_{z 3} u_{3}^{2} \\
& +f_{z 12} u_{1} u_{2}+f_{z 13} u_{1} u_{3}+f_{z 23} u_{2} u_{3}
\end{aligned}
$$

$u_{1}, u_{2}$ and $u_{3}$ are the varying voltages and $f_{x i j}, f_{y i j}$ and $f_{z i j}$ are spacial functions in $x, y$ and $z$. Discrete values of these functions will be computed in a $x, y$ and $z$ grid points using the $3 D$ simulator and stored in a database and a quadratic interpolation is used to evaluate these functions in an arbitrary $(x, y, z)$ point inside the parallelipedic workspace. Using this 
numeric model, the SDDM becomes:

$$
\left[\begin{array}{c}
\dot{x} \\
\dot{y} \\
\dot{z}
\end{array}\right]=\frac{1}{6 \pi \nu R}\left[\begin{array}{l}
F_{D_{x}}(U) \\
F_{D_{y}}(U) \\
F_{D_{z}}(U)
\end{array}\right]
$$

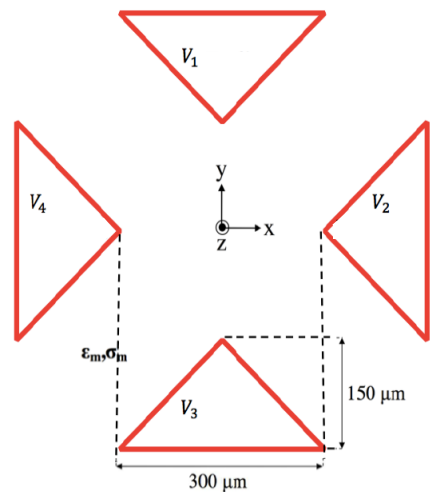

Fig. 5. Geometry of the electrodes and applied voltages: definition of control parameters $u_{x}$ and $u_{y}$.

Consequently, the computation time is reduced and few arithmetic iterations are executed in a very short time, even with the interpolation procedure. Indeed, $60 \mathrm{CPU}$ clock cycles are needed to compute the 3 components of the dielectrophoretic force in a grid point, and $270 \mathrm{CPU}$ clock cycle in an interpolated position. Thus if we consider that the microbead's time response is $3 \mathrm{~ms}$ and for a successful tracking 5 control sequence are generated, a controller card with $1 \mathrm{M} \mathrm{Hz}$ clock takes $0.2 \mathrm{~ms}$ to compute the dielectrophoretic force using the SDDM.

\section{B. 3D inverse dynamic model}

The behavior of a micro-bead in a dielectrophoretic system is characterized by its high dynamics as presented above and the nonlinearity of the generated force with respect to the applied voltages as shown in the equation (11). The analytic inversion of the SDDM (11) is not possible due to the strong coupling between the control variables $u_{1}, u_{2}$ and $u_{3}$ and the generated force. One way to solve this problem is to use the Newton-Raphson numeric method which is able to find the values of the control variables with respect to a required value of the force. Newton-Raphson is a method for finding successively better approximations to the roots of a real-valued functions. By sampling the SDDM (11) and knowing the trajectory $[\hat{x}(t), \hat{y}(t), \hat{z}(t)]$ with respect to the time we are able to compute the appropriate control variable $U(t)$ using the Newton-Raphson method as illustrated in the figure 6:

By sampling the dynamic equation (11) using a sampling period $T$ we obtain:

$$
\left[\begin{array}{c}
\hat{x}_{k+1} \\
\hat{y}_{k+1} \\
\hat{z}_{k+1}
\end{array}\right]=\frac{T}{6 \pi \nu R}\left[\begin{array}{c}
F_{D_{x}}\left(U_{k}\right) \\
F_{D_{y}}\left(U_{k}\right) \\
F_{D_{z}}\left(U_{k}\right)
\end{array}\right]+\left[\begin{array}{c}
x_{k} \\
y_{k} \\
z_{k}
\end{array}\right]
$$

where $\hat{x}_{k+1}, \hat{z}_{k+1}$ and $\hat{z}_{k+1}$ are the next trajectory point at the date $k T$. Applying the Newton-Raphson method to this

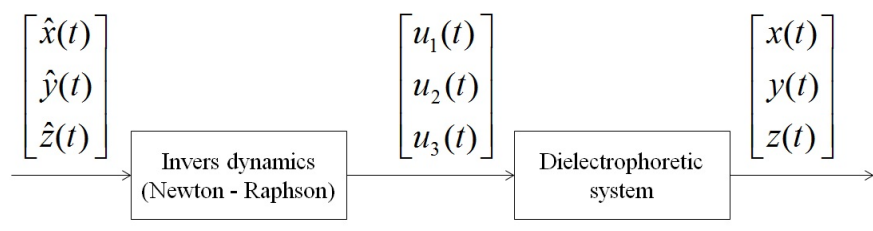

Fig. 6. The Newton-Raphson method is used to find the control variables $u_{1}, u_{2}$ and $u_{3}$

model consists in finding iteratively a series of $u_{1} u_{2}$ and $u_{3}$. At the date $k T$ we have:

$$
U_{k+1}=U_{k}-J^{-1}\left(U_{k}\right)\left[\begin{array}{c}
f_{x}\left(U_{k}\right) \\
f_{y}\left(U_{k}\right) \\
f_{z}\left(U_{k}\right)
\end{array}\right]
$$

where $U_{0}$ are the last computed control variable, $J$ is the Jacobian matrix:

$$
J=\left[\begin{array}{ccc}
\frac{\partial f_{x}}{\partial u_{1}} & \frac{\partial f_{x}}{\partial u_{2}} & \frac{\partial f_{x}}{\partial u_{3}} \\
\frac{\partial f_{y}}{\partial u_{1}} & \frac{\partial f_{y}}{\partial u_{2}} & \frac{\partial f_{y}}{\partial u_{3}} \\
\frac{\partial f_{z}}{\partial u_{1}} & \frac{\partial f_{z}}{\partial u_{2}} & \frac{\partial f_{z}}{\partial u_{3}}
\end{array}\right]
$$

and

$$
\begin{aligned}
& f_{x}(U)=F_{D_{x}}(U)-6 \pi \nu R\left(\hat{x}_{k+1}-x_{k}\right) \\
& f_{y}(U)=F_{D_{y}}(U)-6 \pi \nu R\left(\hat{y}_{k+1}-y_{k}\right) \\
& f_{z}(U)=F_{D_{z}}(U)-6 \pi \nu R\left(\hat{z}_{k+1}-z_{k}\right)
\end{aligned}
$$

The iterations clasically stops when:

$$
\begin{aligned}
& \left\|u_{1_{l+1}}-u_{1_{l}}\right\| \leqslant \delta_{u} \quad \text { and } \quad\left\|u_{2_{l+1}}-u_{2_{l}}\right\| \leqslant \delta_{u} \quad \text { and } \\
& \left\|u_{3_{l+1}}-u_{3_{l}}\right\| \leqslant \delta_{u}
\end{aligned}
$$

where $\delta_{u}$ is an error threshold.

\section{EXPERIMENTATIONS AND RESULTS}

\section{A. Experimental set-up}

In an experimental point of view, the main challenge is to build a control loop able to guarantee a high frequency calculation which requires to optimize the position measurement, the controller, the voltages generator. In micro-scale the most used position sensor is the camera. Thus using vision as feedback,the camera must be a high speed acquisition camera with a high speed communication protocol. As for the voltage generator, the digital analogical converter must have a very short latency (response time) controlled via also a fast communication protocol. These conditions direct us to a very limited choice. Among the different existing solutions we chose for the position sensor the "Photonfocus" camera with Camera Link communication protocol,capable to acquire more than 1000 ips (images per second), in ROI mode (Region of Interest). The frame is grabbed using a PCI (Peripheral Component Interconnect) frame grabber, and the communication time in real time is less then $10 \mu \mathrm{s}$ also in ROI mode. As for the voltages generation we use the 
National Instruments "analog output NI PCI - 6733" with a response time less then $1 \mu s$ using PCI interface. Thus to connect the camera and the voltages generator to the hight speed real time control unit, the controller must have a Camera Link frame grabber attached to a $P C I$ port and an other PCI port for the NI module. The most practical solution is to use the real time Unix. By installing a real time patched kernel in a unix distribution we can create periodic tasks with a deterministic period less than $1 \mu \mathrm{s}$. Thus using a classic $P C$, having at least $2 P C I$ slots we can establish a high speed control system capable to communicate with the camera and the voltages generator. The diagram presented in the figure 7 illustrates the closed loop chain.

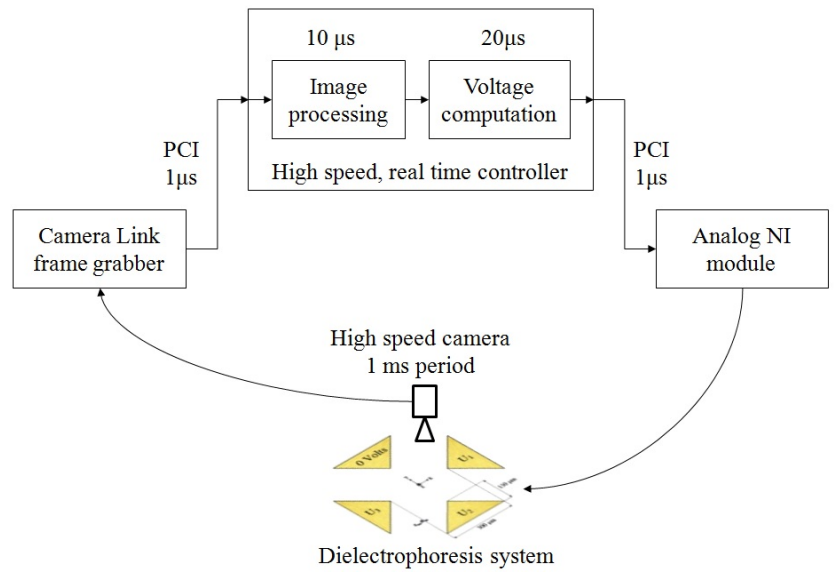

Fig. 7. The closed loop chain. It illustrates each part of the loop with the time taken to complete the chain.

To verify the time taken to measure the position and apply the voltages, we will consider the time taken by each elements of the loop. Starting by measuring the position, a simple image processing algorithm is used to compute the micro-bead's position. It consist of computing the barycenter of the thresholded image as the micro-bead is the only object in the ROI. The execution time of this algorithm depends on the size of the image. If we consider that the ROI is a $256 \times 256$ pixels window, the measured time to compute the micro-bead's position is $10 \mu \mathrm{s}$. The second element in the chain is the control variable (voltages) computation. Using the SDDM presented above, the measured time taken by the computer is $20 \mu \mathrm{s}$. The communication measured between the camera and the controller and the analog output is less than $1 \mu \mathrm{s}$. Thus the time from acquiring the image and applying the voltages is about $30 \sim 40 \mu \mathrm{s}$. The camera frequency is $1 \mathrm{kHz}$, so the controller sampling time is fixed to $1 \mathrm{~ms}$. Between two image acquisitions, the delay between the acquired image and the applied voltages is very small compared to the controller period, thus we can guarantee that each $1 \mathrm{~ms}$ a new voltages are applied with respect to a new measured position.

\section{B. Experimentations}

In the experimental setup the electrodes presented in the figure 8 are used. The electrodes are submerged in the

\begin{tabular}{|c|c|c|}
\hline physical parameters & notations & values \\
\hline vacuum permittivity & $\epsilon_{0}$ & $8,85 \cdot 10^{-12} \mathrm{CV}^{-1} \mathrm{~m}^{-1}$ \\
\hline particle permittivity & $\epsilon_{p}$ & $8,4 \cdot \epsilon_{0}$ \\
\hline particle conductivity & $\sigma_{p}$ & $10^{-12} \mathrm{Sm}^{-1}$ \\
\hline medium permittivity & $\epsilon_{m}$ & $24.9 \epsilon_{0}$ \\
\hline medium conductivity & $\sigma_{m}$ & $1.35^{-11} \mathrm{Sm}^{-1}$ \\
\hline medium volumlic density & $\mathcal{R}_{m}$ & $789 \mathrm{Km}^{-3}$ \\
\hline frequency & $f$ & $10 \mathrm{KHz}$ \\
\hline Clausius-Mossotti & $\operatorname{Re}[\mathrm{K}(\omega)]$ & -0.42 \\
\hline
\end{tabular}

TABLE I

PhysicAl PARAMETER USED IN THE CONTROLLER.

ethanol instead of water in order to reduce the electrolyze effect. The physical parameters used in the SDDM are presented in the table I

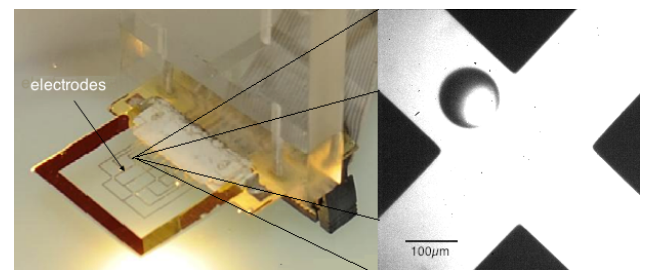

Fig. 8. The electrodes used in the experiments. The microbead's radius is $40 \mu \mathrm{m}$

\section{Discussions}

The experiments show that the dielectrophoretic system is capable of transport particle with high speed $\left(1860 \mu \mathrm{ms}^{-1}\right)$ using closed loop control (fig.9). However, when choosing a faster trajectory reference $\left(5600 \mu \mathrm{ms}^{-1}\right)$, the reference velocity is greater than the maximum velocity (fig.10). The controller computes the optimal voltages enabling the maximum velocity. The bead is following the trajectory with a dynamic error. The particularity of this system is that the velocity is not limited by the voltage generators. Indeed, because of the non-linear behavior of the dielectrophoretic force, maximum velocity is not reached for the maximum voltage, but for an optimal voltage lower than the saturation voltage of our voltage generator. The maximal velocity is defined by the electrodes geometry and the radius of the microbead.

\section{CONCLUSION}

At the micro and nanoscales, robotic manipulation can be based on non contact forces able to induce trajectory where inertia can be neglected. This "No Weight Robots -NWR" can induce high speed trajectory which could of interest in high throughput micro-nano-assembly. This paper has proposed a high speed closed loop control methods based on vision based control. The controller is based on a non-linear model of the system optimized to be computed in less than $1 \mathrm{~ms}$. Experiments show the performance of the trajectory control in high speed velocity (greater than $1000 \mu m . s^{-1}$. Future works will be focused on the manipulation of smaller components dedicated to nanosystems. 

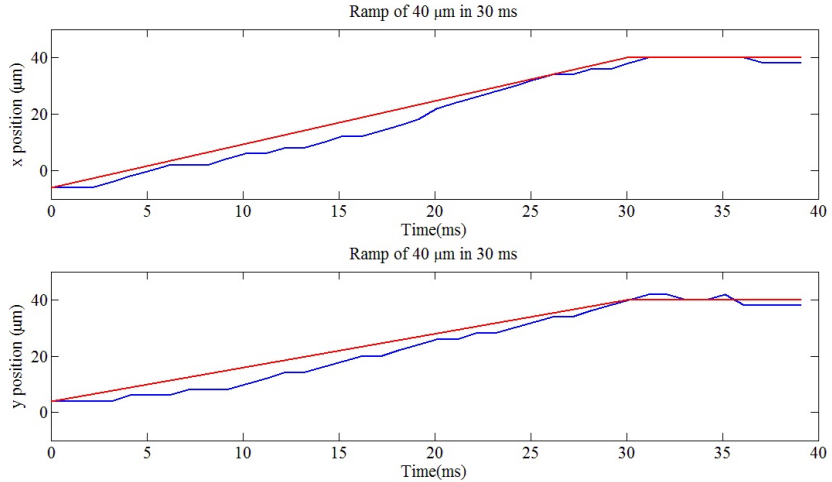

Fig. 9. A $x=y$ ramp trajectory from 0 to $40 \mu m$ during $30 m s$. This figure illustrates a fast controlled trajectory.
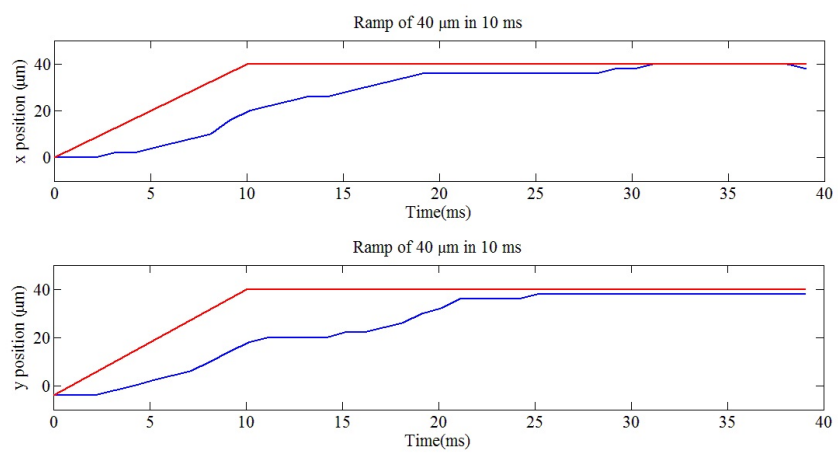

Fig. 10. A $x=y$ ramp trajectory from 0 to $40 \mu m$ during $10 \mathrm{~ms}$. This figure illustrates the saturation of the system. The controller computes the optimal voltages, but the reference trajectory is impossible to track.
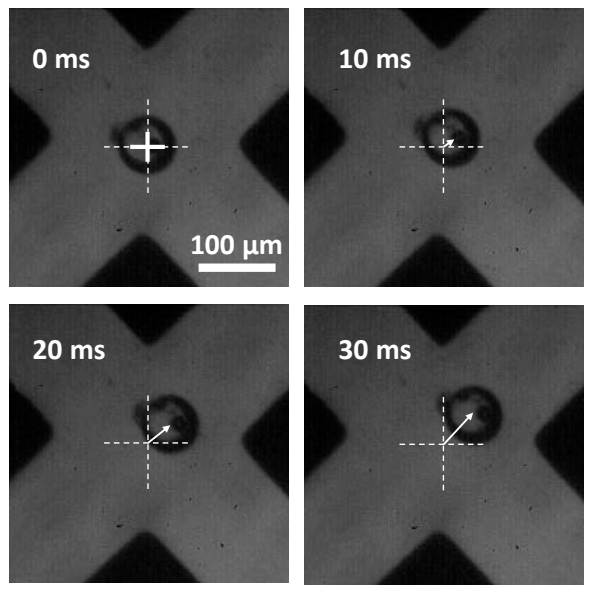

Fig. 11. Examples of photos captured by the camera during the trajectory control reported in figure 9. The microbead radius is $40 \mu \mathrm{m}$, the reference trajectory is a $x=y$ trajectory (see video enclosed).

\section{ACKNOWLEDGMENT}

This work is supported by the european project FP7 FoF FAB2ASM (contract FoF-NMP-2010-260079) (http://fab2asm.eu/); by the Labex ACTION project (contract "ANR-11-LABX-01-01") and by the French RENATECH network and its FEMTO-ST technological facility.

\section{REFERENCES}

[1] G. C. Devol, "Programmed article transfer," USA Patent 2988 237, June 13, 1961.

[2] J. D. Schutter and H. V. Brussel, "Compliant robot motion . a formalism for specifying compliant motion tasks," The int. Journal of Robotics Research, vol. 7, no. 4, pp. 3-17, 1988.

[3] J-P.Bacher, C.Joseph, and R.Clavel, "Flexures for high precision robotics," Industrial Robot: An international Journal, vol. 29, no. 4, 2002.

[4] M. Richard and R. Clavel., "Concept of modular flexure-based mechanisms for ultra-high precision robot design," Mechanical Sciences, vol. 2, pp. 99-107, 2011.

[5] G. Hirzinger, M. Fischer, B. Brunner, R. Koeppe, M. Otter, M. Grebenstein, and I. Schäfer, "Advances in robotics: The dlr experience," The International Journal of Robotics Research, vol. 18, p. 1064, 1999.

[6] G. Schreiber, A. Stemmer, and R. Bischoff, "The fast research interface for the kuka lightweight robot." in IEEE ICRA 2010 Workshop on Innovative Robot Control Architectures for Demanding (Research) Applications, Anchorage, May 2010, p. 2010.

[7] KUKA. [Online]. Available: www.kuka-roboter.de

[8] B. Kratochvil, M. Kummer, J. Abbott, R. Borer, O. Ergeneman, and B. Nelson, "Octomag: An electromagnetic system for 5-dof wireless micromanipulation," in 2010 IEEE International Conference onRobotics and Automation (ICRA), 2010.

[9] M. kharboutly, A. Melis, M. Gauthier, and N. Chaillet, "2d openloop trajectory control of a micro-object in a dielectrophoresis-based device," in 8th IEEE Int. Conf. on Automation Science \& Engineering, Seoul, Korea,, August 2012.

[10] M. kharboutly, A. Melis, A. Bolopion, N. Chaillet, and M. Gauthier, " $2 \mathrm{~d}$ robotic control of a planar dielectrophoresis-based system," in $3 M$ NANO conference, Xi' an, China, August 2012.

[11] F. Pierrot, V. Nabat, O. Company, S. Krut, and P. Poignet, "Optimal design of a 4-dof parallel manipulator: From academia to industry," IEEE Transactions onRobotics, vol. 25, no. 2, pp. 213 - 224, 2009.

[12] S. Bellakehal, N. Andreff, Y. Mezouar, and M. Tadjine, "Force/position control of parallel robots using exteroceptive pose measurements," Meccanica, vol. 46, no. 1, pp. 195-205, 2011.

[13] C. Pawashe, S. Floyd, and M. Sitti, "Multiple magnetic microrobot control using electrostatic clamping,", Applied Physics Letters, vol. 94, p. $164108,2009$.

[14] S. Floyd, C. Pawashe, and M. Sitti, "Two-dimensional contact and non-contact micro-manipulation in liquid using an untethered mobile magnetic micro-robot," IEEE Transactions on Robotics, vol. 25, no. 6 , pp. 1332-1342, 2009.

[15] I. Ivan, G. Hwang, J. Agnus, M. Rakotondrabe, N. Chaillet, and S. Regnier, "First experiments on magpier: A planar wireless magnetic and piezoelectric microrobot," in IEEE International Conference onRobotics and Automation (ICRA), 2011, pp. $102-108$.

[16] M. Kharboutly, M. Gauthier, and N. Chaillet, "Modeling the trajectory of a micro particle in a dielectrophoresis device." Journal of Appl. Phys., vol. 106, no. 11, pp. 114312 - 114312-7, 2009.

[17] T. Sun and H. Morgan, "Ac electrokinetic micro- and nano-particle manipulation and characterization," Electrokinetics and Electrohydrodynamics in Microsystems, CISM Courses and Lectures, Springer, vol. 530, pp. 1-28, 2011.

[18] A. Kuzyk, "Dielectrophoresis at the nanoscale," electrophoresis, vol. 32 , no. 17, pp. 2307-2313, 2011

[19] F. Arai, K. Yoshikawa, T. Sakami, and T. Fukuda, "Synchronized laser micromanipulation of multiple targets along each trajectory by single laser," Appl. Phys. Lett., vol. 85, 2004.

[20] K. Onda and F. Arai, "Multi-beam bilateral teleoperation of holographic optical tweezers," Optics Express, vol. 20, no. 4, pp. 3633 $3641,2012$.

[21] M. Kharboutly, M. Gauthier, and N. Chaillet, "Modeling the trajectory of a micro particle in a dielectrophoresis device," in IEEE International Conference on Robotics and Automation ICRA 2010, Alaska, USA, May 2010.

[22] M. Kharboutly, M. Gauthier, and N. Chaillet,"Predictive control of a micro bead's trajectory in a dielectrophoresis-based device," in IEEE/RSJ International Conference on Intelligent Robots and Systems, Taipei, Taiwan, oct. 2010.

[23] M. P. Hughes, Nanoelectromechanics in Engineering and Biology. CRC PRESS, 2002. 